\title{
Stimulus change effects on matching to sample performance'
}

Pigeons, maintained on a matching to sample procedure, were exposed to a change in the constant stimuli of the experimental environment. Stimulus change produced lower response rates and decreased matching accuracy.

Several studies have shown that a change in the constant stimuli of an experimental environment may disrupt performance. Azrin (1958) found that a change in the constant stimuli disrupted fixed interval performance in humans. Holz \& Azrin (1963) showed that stimulus change disrupted differential reinforcement of low rate performance of pigeons. In the present experiment, the effects of stimulus change on matching to sample performance were investigated with regard to effects on both rate and accuracy of performance. Subjects

The Ss were two adult male homing pigeons maintained at approximately $80 \%$ of their free feeding weight. Apparatus

The experimental chamber was a three key pigeon box similar in design to that described by Ferster (1960). The sample stimuli were always presented on the center key and the matching and nonmatching stimuli were presented on the two side keys. The chamber could be illuminated by two red house lights located in the upper two corners of the response panel. Programming and recording were accomplished automatically by a system of switching relays and timers.

\section{Procedure}

The Ss performed on the matching to sample procedure until stable behavioral performances were obtained. A stimulus consisting of three vertical dots and a stimulus consisting of three horizontal dots were the two sample stimuli. The two samples were presented in a nonsystematic order. A response on the center key produced the matching and nonmatching stimuli on the two side keys. The two stimuli were presented nonsystematically on the right and left keys. The sample stimulus remained illuminated with the matching and nonmatching stimuli. A correct matching response resulted in a $0.5 \mathrm{sec}$. presentation of the magazine light during which the keys were unilluminated. Correct matches were reinforced with a 4-sec. access to grain on a FR 25 schedule. An error produced a 30 sec. time out period during which the keys were not illuminated and responding had no programmed consequences. The house lights remained unilluminated during the training sessions.

Stimulus change was effected by illuminating the house lights. Each $S$ received several stimulus change

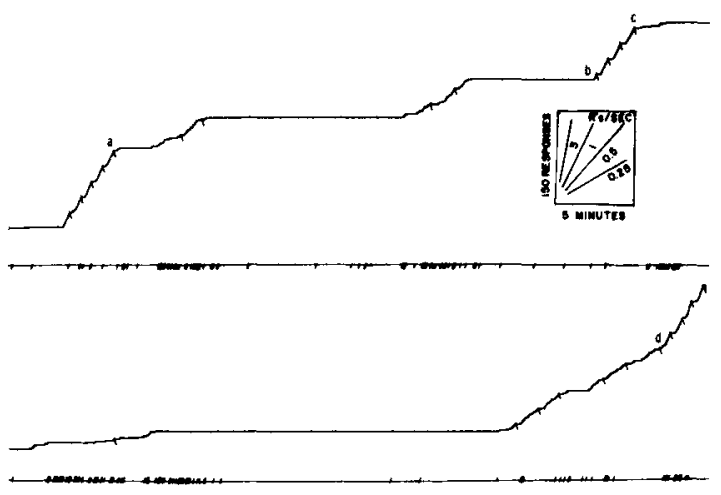

Fig. 1. Cumulative response record for one subject (M5). Correct matching responses are represented by steps of the recording pen. Pips of the recording pen indicate reinforcements. Pips of the event pen indicate matching errors.

probes during three different sessions, the first two spaced 13 days apart and the second two spaced four days apart. During stimulus change probe sessions the house lights were unilluminated during parts of the session in order to have a comparison within a single session of performance under training and stimulus change conditions.

\section{Results and Discussion}

For both sis on all three probe sessions matching to sample performance was severely disrupted by the conditions of stimulus change. Long pauses and low response rates occurred and accuracy was impaired.

Figure 1 presents a cumulative record for one S. Labels $a$ and $c$ of Fig. 1 indicate the introduction of the change in constant stimuli. The labels $b$ and $d$ indicate the reinstatement of training conditions. The typical FR performance was disrupted and long pauses occurred. Both periods of stimulus change were characterized by a lowering of rate and an increase in errors. Response rate (correct and incorrect responses combined) fell from 19 responses per min. to 8 responses per min. and accuracy fell from $96 \%$ to $62 \%$ when the constant stimuli were changed. With the reinstatement of training conditions (Fig. 1, b \& d) the pre-stimulus change performance immediately returned.

Accuracy of performance was recorded separately for responses to the sample stimulus consisting of vertical dots and responses to the stimulus consisting of horizontal dots. Figure 2 shows the probability of a 


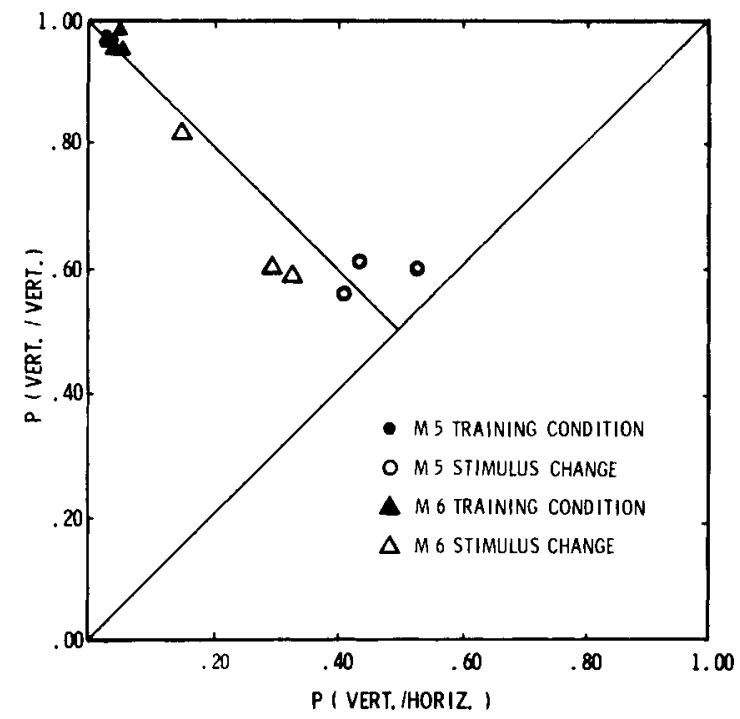

Fig. 2. The probability of correct matches to the vertical dots stimulus as a function of the probability of incorrect matches to the horizontal dots stimulus.

correct match to the vertical dots sample L $\mathbf{P}$ (vertical/ vertical)? as a function of an incorrect match to the horizontal dots sample [ $\mathbf{P}$ (vertical/horizontal)]. The coordinates $(1.0,0.0)$ represent perfect performance; the coordinates $(0.5,0.5)$ represent chance performance. In Fig. 2, performance of both Ss under training conditions is represented by solid circles and triangles.
Performance for the stimulus change conditions is represented by the open circles and triangles. The figure indicates that there was a drop in accuracy almost to chance level under stimulus change conditions. The training condition performances obtained during probe sessions were Identical to performances obtained on prior training sessions. For M6 the disruption was not severe on the third stimulus change session.

Figure 2 allows one to assess response bias as well as accuracy of performance. Divergence from the line between coordinates $(1.0,0.0)$ and $(0.5,0.5)$ indicates the degree of response bias. Performance having coordinates $(1.0,1.0)$ for example would indicate complete response bias towards the vertical dots stimulus, regardless of the sample. The figure shows that the Ss did not develop an appreciable bias towards one stimulus as a result of the disruptions of stimulus change.

The results indicated that stimulus change decreased both rate and accuracy of matching performance but did not increase response bias of the Ss.

\section{References}

Azrin, N. H. Some effects of noise on human behavior. $J$. exp. Anal. Behav., 1958, 1, 183-200.

Ferster, C. B. Intermittent reinforcement of matching to sample in the pigeon. $J$. exp. Anal. Behav., 1960, 3, 259-272.

Holz, W. C., \& Azrin, N. H. A comparison of several procedures for eliminating behavior. J. exp. Anal. Behav., 1963, 6, 399-406.

\section{Note}

1. The present research was supported by grant NsG-450, National Aeronautics and Space Administration. 